few ionic strengths at the seven temperatures at which measurements were made.

There are little data in the literature on activity coefficient terms of weak bases at low ionic strengths. However, if the terms at high ionic strengths are extrapolated to lower strengths, the results of Randall and Failey [4], Harned and Robinson [5], and Harned and Mannweiler [6] obtained by electromotive-force methods and Weil and Morris [7], who used spectrophotometric methods, are in agreement with our results.

\section{Heat of Dissociation}

If one now plots the $-\log K_{a}$ values for each temperature as a function of $1 / T$, a practically straight line is obtained as shown in figure 7 . Thus the heat of dissociation can be assumed to be practically constant, and $\Delta \mathrm{H}$ may be calculated according to eq 8

$$
d \log K / d T=\Delta H / 2.3 R T^{2},
$$

in which $\Delta \mathrm{H}$ represents the molar changes of heat content for the dissociation, $R$ is the gas constant 8.3144 joules $\operatorname{deg}^{-1}$ mole $^{-1}$, and $T$ is the temperature in degrees Kelvin (degrees Celsius + 273.16). $\Delta \mathrm{H}$ is found to be 19,000 joules $\mathrm{deg}^{-1} \mathrm{~mole}^{-1}$.

\section{Basic Ionization Constant, $K_{b}$}

One may derive the basic ionization constant, $K_{b}$, from the usual relationship

$$
K_{b}=K_{w} / K_{a},
$$

in which $K_{v}$ is the activity product for water. For

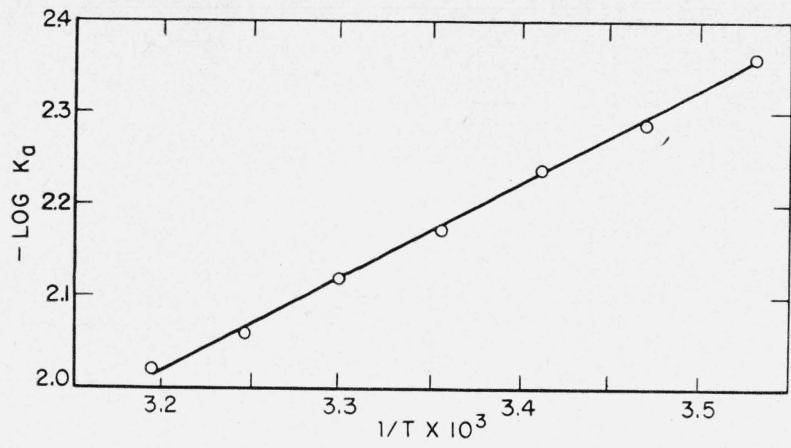

FiguRE 7. $\mathrm{p} K_{a}$ values at temperatures from $10^{\circ}$ to $40^{\circ} \mathrm{C}$ plotted as a function of $1 / T$.

example, at $25^{\circ} \mathrm{C} K_{b}$ is equal to $10^{-14} /\left(6.6 \times 10^{-3}\right)$, which yields a value of $1.6 \times 10^{-11} . \quad \mathrm{p} K_{b}$ is then approximately 11.8 .

\section{References}

[1] Elizabeth E. Sager, Harry J. Keegan, and S. F. Acree, J. Research NBS 31, 323 (1943) RP1569.

[2] Elizabeth E. Sager, Marjorie R. Schooley, and S. F. Acree, J. Research NBS 31, 197 (1943) RP1558.

[3] Elizabeth E. Sager, Marjorie R. Schooley, Alice S. Carr, and S. F. Acree, J. Research NBS 35, 521 (1945) RP1686.

4] M. Randall and C. F. Failey, Chem. Rev. 4, 27k (1927).

[5] H. H. Harned and R. A. Robinson, J. Am. Chem. Soc. 50, 3157 (1928).

[6] H. H. Harned and G. E. Mannweiler, J. Am. Chem. Soc. 57, 1873 (1935).

[7] I. Weil and J. C. Morris, J. Am. Chem. Soc. 71, 3123 (1949).

Washington, August 14, 1950

\title{
A New Method of Radioactive Standard Calibration
}

\author{
By Howard H. Seliger
}

\begin{abstract}
By proper arrangement of experimental observations and statistical analysis it has been possible to make Geiger-counter measurements of different activity samples independent of intermittent disturbing effects. The results of 5,328 individual measurements, involving more than $1.4 \times 10^{8}$ counts made by using a Latin square arrangement, are shown to have an error distribution identical with that expected from the statistical nature of the disintegration process. A completely worked out example of a calibration procedure is given, in which it has been possible to achieve a higher degree of accuracy in only 30 percent of the original counting time.
\end{abstract}

\section{Introduction}

Anyone who has had occasion to make Geigercounter measurements on radioactive isotopes has found at times that his results were not self-consistent; or more precisely, that readings taken at time $A$ could not be duplicated within the expected Poisson deviation at time $B$. Sometimes upon further investigation it was found that these variations were due to fluctuations in the electronic voltage supply, slight changes in sensitivity of the Geiger counter itself, or other transient effects. Most often, if it were not too time-consuming, the original data were discarded and the measurements repeated, this time with the fingers crossed. In this paper a procedure for arranging and analyzing a series of measurements is described that eliminates intermittent disturbing effects and that permits a determination to be made as to whether the variance of the set of readings is a reasonable one to expect on the basis of a Poisson distribution of counts. The end result is that all readings of a set are treated 
as though they were taken at the same time with the same equipment.

\section{Part 1 of the Solution - the Chi Square Test}

If we know that radioactive disintegrations follow a Poisson law, we can predict the variation within which individual values should lie. Our problem is to determine the probability that the variation of an observed set of readings is a reasonable one to expect if the variations are due only to the randomness of the disintegration process. This probability distribution of the ratio of the observed variation to the expected variation has been calculated and is known as the Chi square distribution. ${ }^{1}$ The Chi square statistic is defined as the sum of squares of the deviations divided by the mean, or

$$
\chi^{2}=\frac{\sum_{i}\left(X_{i}-\bar{X}\right)^{2}}{\bar{X}}
$$

where $X_{i}$ is the observed counts per reading, $\bar{X}$ is the expected, or average, counts per reading and $\Sigma$ denotes the summation over all the readings.

Then $\sum_{i}\left(X_{i}-\bar{X}\right)^{2}$ is the sum of the squares of the deviations from the mean count, and therefore an estimate of $n \sigma^{2}$ calculated from the individual counts. When the measurements do follow a Poisson law, the average count is known to be an estimate of $\sigma^{2}$ for the measurements. Thus the expected value of this ratio (eq 1) when the data follow Poisson's law is $n$. This is, however, subject to statistical variations. Tables are available showing for various $n$ the probability limits for this ratio (see footnote 1 ).

Let us now apply this simple test to a set of consecutive readings taken with a Geiger counter and scaler, with a constant activity source. All readings are taken for a constant time interval, which does not enter into the calculations.

\begin{tabular}{|c|c|c|c|}
\hline $\begin{array}{l}\text { Reading } \\
\text { number }\end{array}$ & $\begin{array}{l}\text { Total } \\
\text { counts }\end{array}$ & $\begin{array}{l}\text { Reading } \\
\text { number }\end{array}$ & $\begin{array}{c}\text { Total } \\
\text { counts }\end{array}$ \\
\hline $\begin{array}{l}1 \\
2 \\
3 \\
4 \\
5\end{array}$ & $\begin{array}{l}14,805 \\
15,378 \\
15,021 \\
15,040 \\
15,335\end{array}$ & $\begin{array}{l}6 \\
7 \\
8 \\
9 \\
10\end{array}$ & $\begin{array}{l}15,292 \\
15,287 \\
15,398 \\
15,384 \\
14,908\end{array}$ \\
\hline
\end{tabular}

$$
\begin{aligned}
& { }_{1}^{10}\left(X_{i}-\bar{X}\right)^{2}=435,622, \\
& \bar{X}=15,185, \\
& \chi^{2}=28.7 .
\end{aligned}
$$

1 Peters and VanVoorhies, Statistical procedures and their mathematical bases, p. 404 (McGraw Hill Book Co., Inc., New York, N. Y., 1940); Fisher, Statistical methods for research workers, p. 58 (Oliver and Boyd, London, 1944); K. A. Brownlee, Industrial experimentation, 3d ed., p. 39 (British Ministry of Supply, Directorate of Royal Ordnance Factories, 1948).
As we have 10 readings, there are 9 degrees of freedom. If we look up a table of $\chi^{2}$ for $n=9$, we find that the probability of getting a ratio of 28.7 is less than one chance in a thousand. ${ }^{2}$ Therefore, we conclude that the above set of readings do not follow a Poisson distribution, and that data taken with the counter and scaler are not reliable. Conversely, if this ratio came out within the values 14.68 and 4.17 , which are the 10-percent and 90-percent probability limits, respectively, we could conclude satisfactory operation on the part of our equipment and continue with our measurements. Thus even this simple application of the Chi square test is an extremely useful one for checking equipment, and in the following sections we shall show how it and the method of Latin squares are used together for more complicated data analysis.

\section{Part 2 of the Solution - the Latin Square}

The Latin square was originally developed for agricultural field trials. If $K$ different strains of corn are to be compared, the experimental area is subdivided into a checkerboard of $K$ rows and $K$ columns, making available $K^{2}$ plots. The plantings are applied so that each strain appears once in each row and in each column of the $K^{2}$ plots. This requires that there be $K$ plots assigned to each strain of corn. Every row and every column of the area contains a complete set of the $K$ strains under test. Intuitively it may be seen that such an arrangement will be effective in reducing the error of comparisons among the strains since if any one of the columns or rows is more fertile than the others, all $K$ strains obtain this advantage and the relative performance of the several strains is unaffected. If there exist fertility gradients in the area that would ordinarily interfere with the accuracy of the comparisons, the Latin square method of allocating the plantings to the plots has ensured a more nearly equal opportunity for all strains.

A typical 4 by 4 Latin square arrangement is shown in figure 1, a. The four plants of any given strain appear in the four rows and four columns. The four plots of any one strain may in consequence show considerable variation among themselves, and should not be used directly as an estimate of the error in the comparison of the different strains. However, the comparison of the averages for the strains has been made more accurate by insuring that all strains sample the various strips of fertility. Thus differences among rows and columns no longer contribute to the error of the comparison. It is necessary then to find another way of estimating the error of the average for each strain, as the customary method of calculating the dispersion from plots receiving the same strain is no longer applicable. However, before we proceed to outline the new method for calculating the dispersion, let us carry on with the development of the Latin square arrangement. In figure $1, \mathrm{~b}$, is a Latin square arrangement for the Greek letters $\alpha, \beta, \gamma, \delta$. Now let us superpose figure $1, \mathrm{~b}$, upon figure 1 , a to obtain figure $1, \mathrm{c}$. If the

\footnotetext{
${ }^{2}$ K. A. Brownlee, Industrial experimentation, 3d ed., p. 161 (British Ministry of Supply, Directorate of Royal Ordnance Factories, 1948).
} 


\begin{tabular}{|c|c|c|c|}
\hline$A$ & $B$ & $C$ & $D$ \\
\hline$C$ & $D$ & $A$ & $B$ \\
\hline$D$ & $C$ & $B$ & $A$ \\
\hline$B$ & $A$ & $D$ & $C$ \\
\hline
\end{tabular}

\begin{tabular}{|c|c|c|c|}
\hline$\alpha$ & $\beta$ & $\gamma$ & $\delta$ \\
\hline$\delta$ & $\gamma$ & $\beta$ & $\alpha$ \\
\hline$\beta$ & $\alpha$ & $\delta$ & $\gamma$ \\
\hline$\gamma$ & $\delta$ & $\alpha$ & $\beta$ \\
\hline
\end{tabular}

(a)

(b)

\begin{tabular}{|l|l|l|l|}
\hline$A \alpha$ & $B \beta$ & $C \gamma$ & $D \delta$ \\
\hline$C \delta$ & $D \gamma$ & $A \beta$ & $B \alpha$ \\
\hline$D \beta$ & $C \alpha$ & $B \delta$ & $A \gamma$ \\
\hline$B \gamma$ & $A \delta$ & $D \alpha$ & $C \beta$ \\
\hline
\end{tabular}

(c)

Figure 1. General Latin square arrangement.

a, Latin square for strains A, B, C, D; b, Latin square with Greek letters; c, superposition of b upon a, subject to the same restricting conditions.

four alpha plots in figure 1 , c, are examined, it will be seen that these four plots contain a representative plot from every row and every column and every strain, the strains still being the roman letters A, B, C, D. The same is true for the beta, gamma and delta plots. The averages for these Greek sets therefore should show good agreement, and the variation among these four averages does in fact provide the estimate of error relevant to the comparison of the averages for the four strains. In the same manner, Arabic numerals can be assigned to the plots, so that the four plots marked 1 in figure 2 contain representatives from every row, column, strain, and Greek letter. This is also true for the plots marked 2, 3, and 4 . These four sets also should agree and can be used as a further estimate of the experimental error. The Latin square shown in figure 2 is now unique, or completely orthogonal, in that this is the only way in which the individual simple Latin squares composed of Latin letters, Greek letters, and Arabic numerals, as shown in figure 1 , can be superposed subject to the restricting conditions above. We would expect, therefore, that there should be associated with these classifications as many degrees of freedom as there would be if only one number or letter appeared in each of the 16 squares.

It is customary to summarize the statistical computations for experimental arrangements of this type in a tabulation of the analysis of variance of the five classifications that have been enumerated. They are

\begin{tabular}{|c|c|}
\hline & $\begin{array}{l}\text { Degrees of } \\
\text { freedom }\end{array}$ \\
\hline Variation among the four row averages_._ & 3 \\
\hline Variation among the four column averages & 3 \\
\hline letter) averages & 3 \\
\hline $\begin{array}{l}\text { Variation among the four Greek letter } \\
\text { averages }\end{array}$ & 3 \\
\hline $\begin{array}{l}\text { Variation among the four Arabic number } \\
\text { averages }\end{array}$ & 3 \\
\hline
\end{tabular}

As seen with each of these is associated three degrees of freedom (corresponding to the usual $(n-1)$ divisor in computing standard deviations). The total of 15 degrees of freedom is that properly assigned to 16 observations if a standard deviation were computed for the 16 results ignoring all classifications among the plots. Now, not only do the degrees of freedom add up to the correct total, but the sum of the squares of the deviations within each classification, when added up over the 5 classifications will be found equal to one-fourth of the sum of the squares of the deviations of the 16 observations 


\begin{tabular}{|l|l|l|l|}
\hline $\mathrm{A}_{1} \alpha$ & $\mathrm{B}_{2} \beta$ & ${ }^{9} \mathrm{C}_{3} \gamma$ & $\mathrm{D}_{4} \delta$ \\
\hline${ }^{2} \mathrm{~B}_{4} \gamma$ & ${ }^{6} \mathrm{~A}_{3} \delta$ & ${ }^{10} \mathrm{D}_{2} \alpha$ & ${ }^{14} \mathrm{C}_{1} \beta$ \\
\hline${ }^{3} \mathrm{C}_{2} \delta$ & ${ }^{7} \mathrm{D}_{1} \gamma$ & ${ }^{11} \mathrm{~A}_{4} \beta$ & $\mathrm{B}_{3} \alpha$ \\
\hline${ }^{4} \mathrm{D}_{3} \beta$ & ${ }^{8} \mathrm{C}_{4} \alpha$ & ${ }^{12} \mathrm{~B}_{1} \delta$ & $\mathrm{A}_{2} \gamma$ \\
\hline
\end{tabular}

Figure 2. Arrangement of Latin square to indicate sequence of readings.

about the grand average. ('The factor one-fourth arises because the five components have been computed from averages of four, in contrast to the use of individual observations). It is therefore possible to partition the over-all variance into these five portions. Two of these are the rows and the columns, which by experimental arrangement, have been removed from participation in the experimental comparisons. One portion (Latin letters) directly reflects differences among the strains and the remaining two portions (Greek letters and Arabic numbers), yield independent estimates of the experimental error. Completely worked out examples of this form of Latin square will be found in Brownlee (see footnote 1). The net result is that the computation leads to determining the ratio of the mean sum of squares for each classification to the mean sum of squares for error, as defined by Brownlee (see footnote 1 ). This ratio, called $F$, has the expected value of unity in all cases where the classification under examination contributes nothing to the variation of the results, as would have been the case for instance if only one strain of corn had been sown in all 16 plots. The computed value of $F$ will in such a case be expected to fall in the neighborhood of unity, the reasonable limits of variation being determined by the number of degrees of freedom attached to the numerator and denominator of the ratio. The theoretical distribution of the $F$ ratio has been tabulated (see footnote 1) and is an indispensable guide in passing judgment on a particular value of $F$. These tabulations are listed as Tables of Variance Ratio for various significance levels, corresponding to the probability levels in the $\chi^{2}$ test in section II.

\section{Chi Square Test Modified for the Latin Square}

In section II the principle of the Chi square distribution was demonstrated, and in section III the use of the Latin square for the separation of disturbing factors from the error calculations and for the calculation of variance ratios was outlined. The next steps in our sequence are: (1) To apply the Latin square to our particular problem of calibrating unknown sources of different activities against a known standard source; (2) to modify the calculation of the Chi square test to fit the data taken in our Latin square arrangement.

Perhaps the application of the Latin square to our particular problem will be easier to follow if we start with the end result and then discuss the logic of the arrangement as given. Consider again the Latin square in figure 2 . The 16 subdivisions of the large square are numbered consecutively from 1 to 16 in columnar order in the upper left-hand corners. These numbers represent the sequence of the readings taken. The Latin letters A, B, C, and D represent a primary standard source and three unknown sources, respectively. We shall consider the standard source and the three unknown sources as our "strains". As the observed counting rates due to the sources A, B, C, and D are in general different, (due to the fact that the absolute disintegration rates of the sources are different) a variance among the four source averages (Latin letters) is to be expected and therefore should not be included in any error calculations. However, the sums of sets of four readings as for instance the sum of the $\alpha$ 's, include only one reading each of $\mathrm{A}, \mathrm{B}, \mathrm{C}$, and D, and therefore the sum of the $\alpha$ 's theoretically should be equal to the sum of the $\beta$ 's, $\gamma$ 's, and $\delta$ 's. This being the case, we can perform our Chi square calculation on the sums of four readings instead of on the individual readings. Again, from section III, we have the following sets of sums of four readings: Rows; columns; like Latin letters (sources); like Greek letters; like Arabic numbers.

The variation among the sources (Latin letters) is immediately ruled out for the reason already given. From the sequence of the measurements as given by the numbers in the upper left-hand corners of the squares in figure 2 , it is seen that the sums of the columns will be especially sensitive to short-term intermittent disturbing factors of all kinds. Thus the variance among the four column averages should not be included in any error calculation. Moreover, these intermittent fluctuations, if they occur, are analogous to the fertility differences in our agricultural example, so that the relative performance of our four sources should be unaffected (if we consider sums and not individuals).

Now we have remaining three sets of four sums each for the calculation of error. These are the rows, the Greek letters and the Arabic numbers. The next step is to modify our Chi square calculation to fit these data.

We know that in our particular case of radioactive disintegrations $\sigma^{2}$, the standard deviation squared of our Poisson distribution, is equal to $N$, where $N$ is the total counts involved. The estimate of $n \sigma^{2}$ can be calculated from the three sets of four sums. In this case there are 3 degrees of freedom from each set, making a total of 9 degrees of freedom.

Table 1 shows a complete set of data taken in the calibration of three unknown sources B, C, and D against a laboratory standard source, A. In order to indicate clearly the sequence of the readings and 
TABLE 1. Data as taken in an actual calibration of sources.

\begin{tabular}{|c|c|c|c|c|c|}
\hline READ & \multirow{2}{*}{$\begin{array}{c}\text { NO. } \\
\text { SAMPLE }\end{array}$} & TOTAL & TOTAL & \multicolumn{2}{|c|}{ COUNTS/SEC } \\
\cline { 5 - 6 } & & COUNTS & SEC & GROSS & NET \\
\hline I & A & 34480 & 1277 & 27.00 & 26.46 \\
\hline 2 & B & 27364 & 973.1 & 28.12 & 27.58 \\
\hline 3 & C & 25650 & 864 & 29.69 & 29.15 \\
\hline 4 & D & 25774 & 856.8 & 30.08 & 29.54 \\
\hline 5 & B & 27060 & 947.1 & 28.57 & 28.03 \\
\hline 6 & A & 30540 & 1109 & 27.54 & 27.00 \\
\hline 7 & D & 27478 & 914 & 30.06 & 29.52 \\
\hline 8 & C & 25628 & 850 & 30.15 & 29.61 \\
\hline 9 & C & 25614 & 858 & 29.85 & 29.31 \\
\hline 10 & D & 26404 & 878.1 & 30.07 & 29.53 \\
\hline 11 & A & 26082 & 965.4 & 27.02 & 26.48 \\
\hline 12 & B & 25612 & 903.2 & 28.36 & 27.82 \\
\hline 13 & D & 25650 & 864 & 29.69 & 29.15 \\
\hline 14 & C & 25696 & 866.2 & 29.67 & 29.13 \\
\hline 15 & B & 25654 & 902.1 & 28.44 & 27.90 \\
\hline 16 & A & 26510 & 980 & 27.05 & 26.51 \\
\hline
\end{tabular}

the method of summation, these data have been arranged in the form of a Latin square in table 2 .

The analysis is performed in the following manner:

1. Calculate the average row sum of the four sets of row sums. Notice here that in order to do this, all 16 readings are included, and this grand sum is divided by 4 . Therefore the average row sum is equal to the average Greek letter sum and also to the average Arabic number sum. Let us call this value

$$
\overline{\Sigma(4)}=113.18 \text {. }
$$

2. Calculate the sum of squares of the deviations of the individual row sums from the average sum of four.

$$
\left(0.23^{2}+0.06^{2}+0.13^{2}+0.30^{2}=0.1634\right) .
$$

3. Calculate the sum of squares of the deviations of the individual Greek letter sums from the average sum of four.

$$
\left(0.25^{2}+0.04^{2}+0.57^{2}+0.36^{2}=0.5186\right) .
$$

4. Calculate the sum of squares of the deviations of the individual Arabic numbers sums from the average sum of four.

$$
\left(0.32^{2}+0^{2}+0.26^{2}+0.06^{2}=0.1736\right) .
$$

\begin{tabular}{|c|c|c|c|c|c|}
\hline & & & & & $\begin{array}{l}\text { ROW } \\
\text { TOTALS }\end{array}$ \\
\hline & 26.46 & 28.03 & 29.31 & 29.15 & 112.95 \\
\hline & Ala & $\mathrm{B}_{2} \beta$ & $\mathrm{C} 3 \mathrm{y}$ & $04 \delta$ & \\
\hline & $\begin{array}{l}27.58 \\
27\end{array}$ & $\begin{array}{l}6 \\
27.00\end{array}$ & $\begin{array}{l}10 \\
29.53\end{array}$ & 29.13 & 113.24 \\
\hline & $\mathrm{B}_{4} \mathrm{Y}$ & $A 3 \delta$ & $\mathrm{D} 2 \alpha$ & $C_{1} \beta$ & \\
\hline & 29.15 & $\begin{array}{l}7 \\
29.52\end{array}$ & "I 26.48 & \begin{tabular}{|l|}
15 \\
27.90
\end{tabular} & 113.05 \\
\hline & $\mathrm{C}_{2} \delta$ & DIY & $A_{4} \beta$ & Bз $\alpha$ & \\
\hline & 4 & 8 & 12 & 16 & \\
\hline & 29.54 & 29.61 & 27.82 & $26.5 \mid$ & 113.48 \\
\hline & D $3 \beta$ & $\mathrm{C}_{4} \alpha$ & $B / \delta$ & $\mathrm{A}_{2} \gamma$ & \\
\hline $\begin{array}{l}\text { COLUMN } \\
\text { TOTALS }\end{array}$ & 112.73 & 114.16 & 113.14 & 112.69 & \\
\hline ARABIC & $1+7$ & $3+5+10+16$ & $5+9+15$ & $2+8+11+13$ & \\
\hline TOTALS & 112.93 & 113.22 & 113.75 & 112.82 & \\
\hline GREEK & $1+8+10+15$ & $4+5+11+14$ & $2+7+9+16$ & $3+6+12+13$ & \\
\hline TOTALS & 113.50 & 113.18 & 112.92 & 113.12 & \\
\hline $\begin{array}{l}\text { LATIN } \\
\text { OR }\end{array}$ & A & B & C & D & \\
\hline $\begin{array}{l}\text { SAMPLE } \\
\text { TOTALS }\end{array}$ & 106.45 & 111.33 & 117.20 & 117.74 & \\
\hline
\end{tabular}

TABLE 2. Data from table 1 arranged in a Latin square to make the summation procedure easier to follow.

5. Sum these three sums of squares.

$$
(0.1634+0.5186+0.1736=0.8556) .
$$

6. We must digress for a moment and consider now the basis for this calculation. The expected standard deviation squared of a number of events that follow a Poisson law is $N$, when $N$ is the total number of events. However, as is seen in table 1, our sample deviations are calculated from observed counting rates. In order that our ratio of observed to expected deviations be a unitless quantity, we must express the sum of squares of the deviations as total counts. This can be done in the following manner:

In each individual measurement we observe at least 25,600 counts, which is 200 message register counts on a scale of 128 . Therefore, we can, with negligible error, assume that for a set of four readings the total number of counts involved is $4 \times 25,600=102,400$. If we divide this value by the average counting rate for a sum of four readings, we get a value with the units of seconds, 
corresponding to the average time per reading. This value squared will, when multiplied by the sum of squares of the deviations that has the units of $[(\text { counts }) /(\text { seconds })]^{2}$, give units of (counts) ${ }^{2}$, the same as our denominator.

Our calculated ratio is now

$$
\chi^{2}=\frac{0.8556 \times\left[\frac{102,400}{113.18}\right]^{2}}{102,400}=6.84
$$

In our table of $\chi^{2}$ we find, for 9 degrees of freedom

$$
\begin{aligned}
& \chi_{.70}^{2}=6.39, \\
& \chi_{.50}^{2}=8.34 .
\end{aligned}
$$

Thus our ratio has greater than a 50-percent probability of occurence on the basis of a Poisson law, and we conclude that the resulting calibrations are perfectly acceptable.

As an illustration of the efficiency of this method in eliminating transient disturbances from the measurements, let us perform an $F$ test on the column totals. The mean square column deviations are $1.4046 / 3$, as here we have 3 degrees of freedom. The mean-square-error deviations are $0.8556 / 9$, since there we used 9 degrees of freedom. Thus the ratio 4.92 , as we can see from tables of Variance Ratio has reached the 5-percent significance level. Evidently in this case the Latin square arrangement was successful in removing this effect from the variance of the sample averages. It is the elimination of these disturbances that accounts for the increased accuracy over the old direct-comparison method and the subsequent 70 percent shorter counting time. This reduction in counting time is due to two factors. First, the number of runs used was reduced. Where previously a standard source and three unknown sources were run alternately, so that for 4 runs on each of the three unknowns the standard would be run 13 times, making a total of 25 runs for the entire set of readings, we now have only 16 runs in which the standard is measured as many times as the unknowns. Second, as the extraneous (nonPoisson) variation was present in the former method, a much larger counting time was necessary than that based on a Poisson distribution in order to obtain the precision called for. The Latin square arrangement permits the counting time to be cut in half, and the precision of the measurements is still greater than in the old method. is

The standard deviation of any of the sums of four

$$
s_{\Sigma}=\left[\frac{0.8556}{9}\right]^{\frac{1}{2}}=.308
$$

The standard deviation of any individual counting rate is

$$
s=\left[\frac{0.8556}{4 \times 9}\right]^{\frac{1}{2}}=0.154
$$

For example, the average counting rates of sources $\mathrm{A}, \mathrm{B}, \mathrm{C}$, and D, which are the objectives of this entire procedure, are, respectively, 26.61, 27.83, 29.30 , and $29.44 \pm .15 \mathrm{c} / \mathrm{s}$. These results can also be calculated as shown in Brownlee (see footnote 1). However, the method as demonstrated is more direct and easier to calculate.

\section{Summary}

In this paper two things have been accomplished. First, by the arrangement of the experimental data in the form of the orthogonal Latin square, thereby eliminating intermittent effects, the accuracy has been increased to such an extent that only 30 percent of the original counting time is required. Second, it is now possible to determine the actual experimental standard deviation of any particular average reading, and in the process of obtaining this standard deviation it is also possible to determine whether or not the readings follow a Poisson law, which is the case when the equipment is operating satisfactorily.

As can be seen, the use of Latin squares for the statistical arrangement of measurements is an extremely powerful tool, by means of which a number of separate effects may be determined or eliminated. It has proved to be extremely efficient in the laboratory, not only in the actual calibration of standards, but in obtaining useful information relative to the long-range operating characteristics of the Geiger counters and associated electronic equipment. The general method as outlined should be applicable to a large variety of other types of measurements, especially where time effects or other disturbing factors are a source of trouble.

The results of 333 such sets of data as shown in tables 1 and 2 have been tabulated and are presented in figure 3, together with their theoretical distribution. As can be seen from the figure, the experimental distribution closely approximates the theoretical one. There is one more point that can be

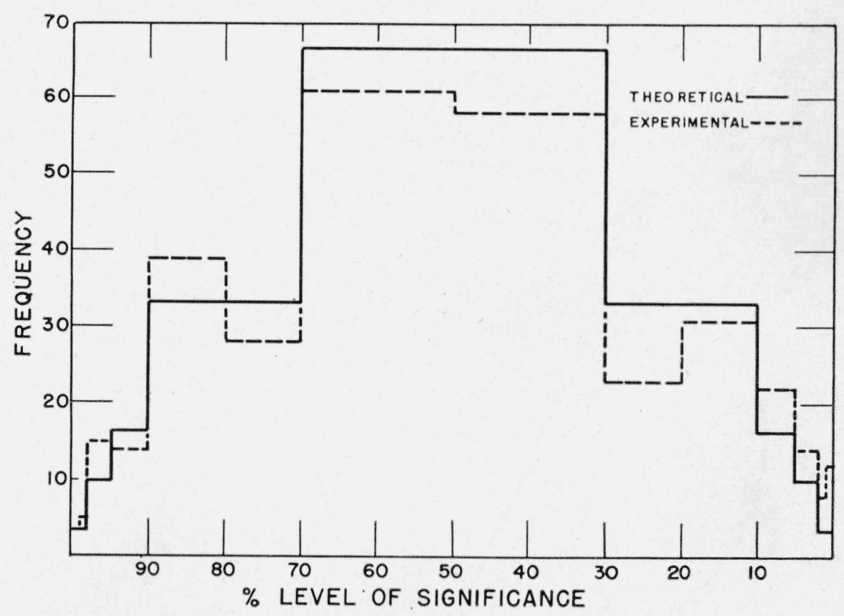

Figure 3. Frequency distribution of 333 sets of Chi square data (broken line) compared with theoretical distribution (solid line). 
made only after the accumulation of such a large amount of data as this. The small peaks at the right of figure 3 indicate a slight tendency for the observed counts to be influenced by effects other than statistical fluctuations. However, it must be remembered that the data include instances where either counters or scaling circuits have gone bad and have evidenced a need for repair or replacement, so that this slight excess is to be expected. It is believed that this is the first time that such a large collection of Chi square values has been obtained from the ratio of the error variance of Latin squares to the expected (Poisson) variance.

It is well known that individual measurements of radioactive disintegrations follow a Poisson law, but the close agreement between the experimental and theoretical curves illustrates one further point: that the Latin square arrangement has been completely successful in eliminating extraneous, nonPoisson fluctuations from the measurements.

The author thanks W. J. Youden and J. M. Cameron, of the Statistical Engineering Section, for their suggestions as to the use of the Latin squares and for many valuable hours of discussion; and Margaret Selgin and Lucy Cavallo, of the Radioactivity Section, who performed the calculations summarized in figure 3 .

Washington, July 26, 1950.

\title{
A Problem in Precision Cam Design
}

\section{By Joseph Blum}

\begin{abstract}
This paper proposes an analytic method for determining the profile of a cam required in a device that demands high precision. The method is applicable whenever the equation for the profile can be obtained by making a less stringent assumption, namely, that the follower makes contact at a fixed point of its extremity. The true profile can then be determined numerically by considering it as the envelope of a family of curves.
\end{abstract}

The design of an X-ray spectrograph often requires that a constant rate of oscillation be maintained in the crystal holder. The crystal rotates through an angle $\beta$ (the angle of travel), reverses its direction and then proceeds with the same angular velocity. This type of motion may be obtained from a cam rotating with constant angular velocity, $\omega$. An analytic method for computing the profile of the cam is presented in this paper which extends, in a certain sense, the application of a paper written by J. B. Friauf; ${ }^{1}$ the notation therein will be preserved to a considerable extent.

\footnotetext{
1 J. B. Friauf, The design of a cam for an X-ray spectrograph, J. Opt. Soc. Am Rev. Sci. Instr. 11, 289 to 296 (1925).
}

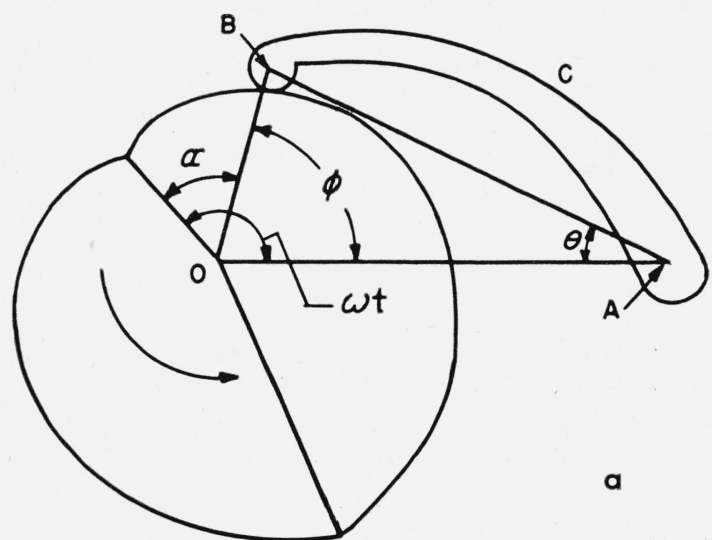

In figure 1, a, $O$ represents the axis about which the cam rotates, $A$ the axis about which the crystal holder rotates, and $B$ the center of the ball at the end of the follower. The follower $A C B$ makes contact with the cam and serves to rotate the crystal holder. The distances $A O$ and $A B$ are equal; denote this common length by $R$. Let $r_{1}$ and $r_{2}$ be, respectively, the least and greatest radii measured from $O$ to the point $B$; let $r$ be the variable radius, $O B$ corresponding to the angle $\alpha$, which $r$ makes with the least radius $r_{1}$.

Denote the time by $t$ and let $t=0$ represent the time when the least radius coincides with the line segment extending from $O$ to $A$. Then the angle

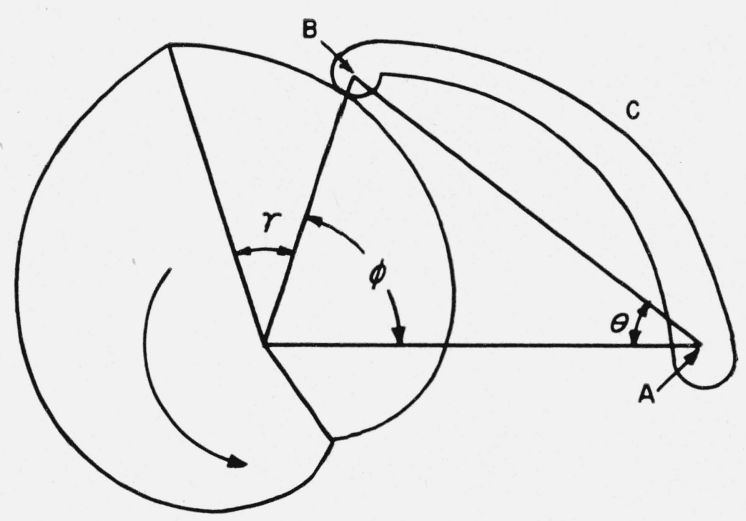

b

Figure 1. 